\title{
Enseñanza de la Cinemática Directa Utilizando LEGO Mindstorms ${ }^{\mathrm{TM}}$
}

\author{
S. González-García ${ }^{\mathrm{a}, 1^{*}}$, G. Loreto-Gómez ${ }^{\mathrm{b}}$, J. Rodríguez-Arce ${ }^{\mathrm{c}}$
}

\author{
${ }^{a}$ Departamento Regional de Computación y Mecatrónica. Escuela de Ingeniería y Ciencias. Región Centro. Tecnológico de Monterrey Campus Morelia Av. Montaña \\ Monarca 1340. Col. Ejido Jesús del Monte. Morelia 58350, Michoacán, México. \\ ${ }^{b}$ Departamento de Ingeniería Mecatrónica, Instituto Tecnológico Superior de Uruapan. Carretera Uruapan-Carapan No. 5555 Col. La Basilia. Uruapan 60015, \\ Michoacán, México. \\ ${ }^{c}$ Facultad de Ingeniería, Universidad Autónoma del Estado de México. Ciudad Universitaria Cerro de Coatepec s/n, Colonia Universidad, Toluca 50110, México.
}

\begin{abstract}
Resumen
El presente trabajo tiene como objetivo mostrar una propuesta metodológica para la enseñanza de la cinemática directa de manipuladores industriales, el cuál es uno de los principales temas de un curso introductorio de robótica para estudiantes universitarios. La técnica utilizada para la enseñanza de la cinemática directa de manipuladores tiene su fundamento en la convención introducida por Denavit y Hartenberg; este método se fundamenta en el análisis de los cuerpos rígidos que constituyen el manipulador y la obtención de parámetros que permitan definir las matrices de transformación homogénea que relacionan el sistema de referencia del efector final con el sistema de referencia de la base del robot. La simulación y la experimentación en el laboratorio pueden ser de gran ayuda para mejorar la comprensión de dichos conceptos. El enfoque propuesto permite a los estudiantes reafirmar sus conocimientos a través de una plataforma de manipuladores modelados en Solidworks ${ }^{\mathrm{TM}}$ y exportados al entorno de MATLAB ${ }^{\mathrm{TM}}$ Simulink $^{\mathrm{TM}}$ utilizando la librería de SimMechanics ${ }^{\mathrm{TM}}$ y verificar su habilidad para resolver un problema de cinemática directa de un manipulador físico construido con el equipo didáctico de LEGO Mindstorms ${ }^{\mathrm{TM}}$ controlado mediante la librería de LEGO Mindstorms $^{\mathrm{TM}} \mathrm{EV} 3$ para Simulink ${ }^{\mathrm{TM}}$.
\end{abstract}

Palabras Clave: Cinemática directa, SimMechanics ${ }^{\mathrm{TM}}$, LEGO Mindstorms ${ }^{\mathrm{TM}}$.

\section{Introducción}

El método tradicional para enseñar los temas fundamentales en un curso introductorio de robótica industrial incluye exposiciones por parte del profesor con algunas sesiones para resolver ejercicios de libros de texto clásicos como (Spong, M. W., et al., 2008). El aprendizaje de los conceptos fundamentales de la robótica como la cinemática, la dinámica, la transformación de coordenadas, la planificación de trayectorias y el diseño de controladores no es una actividad fácil ni motivante para los estudiantes, sin mencionar la dificultad que representa comprender un concepto matemático y relacionarlo inmediatamente con el comportamiento de un robot. Utilizando el método convencional para enseñar los temas generalmente se abordan a través de presentaciones estáticas basadas principalmente en texto y figuras, lecturas en libros de texto y algunas lecciones prácticas en laboratorios que consisten en prácticas guiadas demostrativas con el robot físico que implica el establecimiento de ciertos límites que no permiten a los estudiantes el poder explorar los conceptos por su cuenta. Bajo este esquema, el profesor y los estudiantes no pueden "experimentar" con los conceptos explicados.

El enfoque anterior, ha sido reforzado al integrar el uso de diferentes programas de cómputo que permiten simular el comportamiento de los manipuladores industriales, en el contexto de la enseñanza, una herramienta de simulación

\footnotetext{
* Autor en correspondencia.

Correos electrónicos: sgg@tec.mx (S. González-García),
}

dr.gloreto@hotmai 1 .com (G. Loreto-Gómez), jrodrigueza@uaemex.mx

(J. Rodríguez-Arce) interactiva tiene por objetivo el mostrar un conjunto resumido de conceptos teóricos a través de una interacción en tiempo real. $\mathrm{Su}$ propósito principal es que el profesor y los estudiantes puedan interactuar con los parámetros involucrados en los conceptos explicados y puedan observar el efecto de dicha modificación y cómo afecta al comportamiento global del manipulador analizado. En (Corke, P. I., 2011,) y (Gil, A., et al., 2014) se han desarrollado herramientas en MATLAB para la enseñanza de la robótica a nivel universitario. Una aplicación similar es presentada por (Joshi, S. S. , 2004). La aplicación de este tipo de herramientas de simulación dentro del aula ha permitido aumentar la motivación y aprendizaje por parte de los estudiantes como se demuestra en (Loreto-Gómez et al., 2017). En (Miller, D., et al., 2008) se realiza una buena revisión de la literatura referente a la enseñanza de la robótica.

Por otro lado, el tema de la cinemática directa que corresponde en determinar la posición y orientación del elemento terminal del manipulador conocido como efector final con respecto a su base a través del conocimiento de la posición angular de sus articulaciones, es un tema que requiere por parte de los estudiantes ciertas habilidades matemáticas y, lo que es más importante, una buena percepción espacial de los movimientos que realizan los eslabones del manipulador unidos generalmente a través de articulaciones de tipo rotacional y/o prismática. 
La convención introducida por Denavit y Hartenberg permite simplificar y estandarizar la expresión de la información cinemática para manipuladores de $n$-eslabones a través un conjunto de valores conocidos como los parámetros de Denavit-Hartenberg (Parámetros D-H), a partir de los cuales se pueden obtener las matrices de transformación homogénea que expresan matemáticamente la posición y orientación relativa entre los sistemas de referencia correspondientes a los $n$-eslabones rígidos del manipulador, y que son utilizados para determinar la cinemática directa. Para apoyar la comprensión de estos parámetros en las diferentes configuraciones de manipuladores industriales han sido propuestos en la literatura una variedad de plataformas de simulación, como ejemplo, se encuentran la propuesta desarrollada por (Othayoth, et al., 2017) que cubre los tópicos de la cinemática directa e inversa, en particular, se puede visualizar los parámetros de D-H y cómo afectan la arquitectura de un robot. En (Gil et al., 2014) se presenta una aplicación con simulaciones 3D de robots manipuladores comerciales, la herramienta es utilizada para cubrir temas de la cinemática directa e inversa, la dinámica directa e inversa, generación de trayectorias y programación de robots.

Por otro lado, la enseñanza de los diferentes temas de robots manipuladores son difíciles de complementar con el trabajo experimental debido a que, por una parte se enseñan conceptos teóricos que dan el sustento a su análisis y, por la parte experimental, generalmente se enseñan los comandos básicos para programar el manipulador a efecto de que realice algunas tareas especificadas; para superar el inconveniente anterior, el uso de plataformas configurables se ha propuesto en (Berenguel, et al., 2015) y (Indri, et al., 2013). Sin embargo, desde la perspectiva de los autores, la simulación no toma en cuenta elementos de un manipulador industrial real, siendo en algunas ocasiones complicado para los estudiantes identificar la relación entre el análisis del modelo simulado y la aplicación de conocimientos en un caso práctico.

El utilizar equipo didáctico como LEGO Mindstorms ${ }^{\mathrm{TM}}$ para la etapa practica de un curso se justifica por un lado, debido a que las instituciones educativas en México y Latinoamérica difícilmente tienen posibilidad de contar con un manipulador industrial cuyo costo ronda en los miles de dólares, sin embargo, la adquisición de una plataforma que cuesta algunos cientos de dólares es una inversión que es posible realizar, y por el otro lado, esta plataforma educativa tiene la versatilidad de permitir la construcción de una gran variedad de sistemas mecánicos, en particular, las configuraciones de manipuladores tipo industrial (Berenguel, et al., 2015).

El presente trabajo se encuentra organizado de la siguiente manera: En la sección 2 se presentan los fundamentos teóricos referentes a las transformaciones homogéneas entre sistemas de referencia y el concepto de la cinemática directa. En la sección 3 se muestra la metodología de aprendizaje empleada en un curso introductorio de robótica a nivel universitario para la enseñanza de la cinemática directa, además, se describe la herramienta de simulación y el robot manipulador construido mediante el equipo didáctico de LEGO Mindstorms ${ }^{\mathrm{TM}}$, ambos utilizados en la evaluación realizada a los estudiantes. Finalmente, en la sección 4 se muestran las conclusiones y trabajo a futuro.

\section{Preliminares}

Un manipulador industrial puede modelarse como un sistema de cuerpos rígidos que mantienen cierto movimiento relativo entre ellos y cuyo objetivo es realizar un conjunto de tareas específicas a través de una serie de movimientos que deben realizar cada uno de los elementos que lo constituyen; para cumplir lo anterior es necesario además determinar las relaciones espaciales existentes entre los elementos del manipulador y de los diferentes objetos que se encuentran dentro de su área de trabajo. Los problemas a estudiar por la cinemática en el área de la robótica industrial se agrupan en dos grandes tópicos: El problema de la cinemática directa consiste en determinar la posición y orientación del cuerpo o elemento final del robot con respecto al sistema de referencia base cuando son conocidas las posiciones articulares y las dimensiones geométricas de los eslabones que integran el manipulador; por otro lado, el problema de la cinemática inversa consiste en determinar las posiciones que deben adoptar cada uno de los eslabones que integran el manipulador cuando son conocidas la posición y orientación del elemento final con respecto al sistema de referencia base (Barrientos, A., 2007). En particular, en este trabajo se presenta la experiencia de los autores en la aplicación de una metodología para la enseñanza de la cinemática directa.

Para determinar la cinemática directa de un manipulador industrial se asume como un mecanismo de cadena cinemática abierta formado por $n$ eslabones rígidos unidos entre sí a través de $n$ articulaciones, de forma que cada par articulación-eslabón constituye un grado de libertad (GDL). A cada eslabón se le asocia un sistema de referencia cartesiano y a través de la aplicación de las transformaciones homogéneas se determina las rotaciones y traslaciones relativas entre los distintos eslabones que componen el manipulador.

Para representar la posición y orientación de un objeto se suelen hacer uso de transformaciones geométricas de traslación y de rotaciones sucesivas o encadenadas representadas en coordenadas homogéneas. El uso de coordenadas homogéneas permite tratar todas las transformaciones geométricas como una multiplicación de matrices, la matriz resultante de todas las transformaciones aplicadas es denominada comúnmente como matriz de transformación homogénea, T. Para cualquier secuencia de transformaciones de rotación y de traslación es posible calcular la matriz de transformación homogénea, calculando el producto de las transformaciones individuales.

Una transformación homogénea de traslación corresponde a desplazar un sistema de referencia con respecto a otro sistema de referencia considerado como base. En la figura 1 se muestra una transformación de traslación del sistema de referencia $\{B\}$ cuyos vectores de dirección unitarios son coincidentes con los del sistema de referencia $\{A\}$, el origen del sistema $\{B\}$ se traslada con respecto al sistema $\{\mathrm{A}\}$ mediante la matriz, $\boldsymbol{T}_{t}$. 


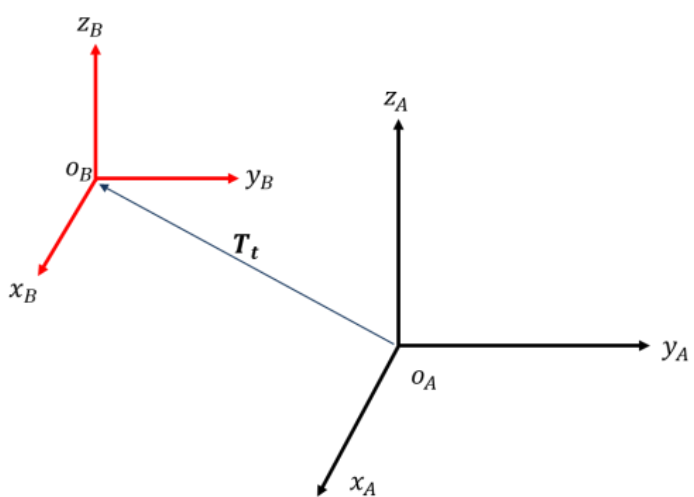

Figura 1: Transformación de traslación.

La matriz de transformación homogénea que representa la transformación de traslación tiene la siguiente forma:

$$
\boldsymbol{T}_{\boldsymbol{t}}\left(t_{x}, t_{y}, t_{z}\right)=\left(\begin{array}{cccc}
1 & 0 & 0 & t_{x} \\
0 & 1 & 0 & t_{y} \\
0 & 0 & 1 & t_{z} \\
0 & 0 & 0 & 1
\end{array}\right)
$$

Análogamente, una transformación homogénea de rotación de un sistema de referencia con respecto a otro base, consiste en rotar sus vectores unitarios de los ejes coordenados. Sea un sistema de referencia $\{B\}$ cuyos vectores de dirección son coincidentes con los de otro sistema $\{\mathrm{A}\}$, los vectores unitarios del sistema $\{\mathrm{B}\}$ se rotan con respecto al sistema $\{\mathrm{A}\}$ mediante una matriz de transformación homogénea si y solo si, el origen de ambos sistemas coincide y la rotación se realiza con respecto a uno de los ejes del sistema base, por lo tanto, se puede rotar con respecto al eje $x$, o al eje $y$ o al eje $z$ como se ilustra en la figura 2 .
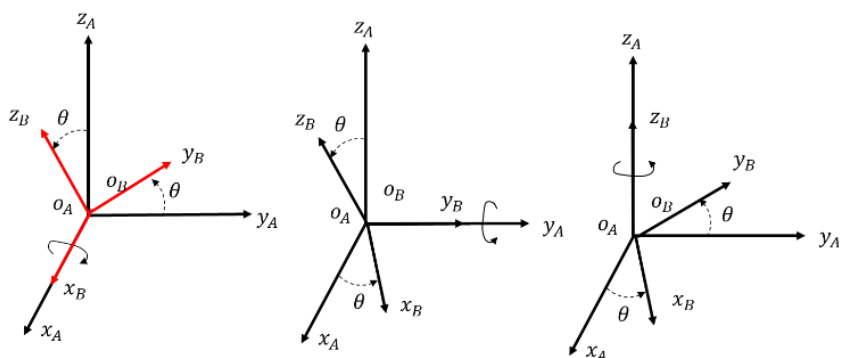

Figura 2: Transformación de rotación con respecto al eje $x$, al eje $y$, eje $z$, respectivamente.

La matriz de transformación homogénea que representa la transformación de rotación con respecto al eje $x$ tiene la siguiente forma:

$$
\boldsymbol{T}(x, \theta)=\left(\begin{array}{cccc}
1 & 0 & 0 & 0 \\
0 & \cos \theta & -\sin \theta & 0 \\
0 & \sin \theta & \cos \theta & 0 \\
0 & 0 & 0 & 1
\end{array}\right)
$$

La matriz de transformación homogénea que representa la transformación de rotación con respecto al eje $y$ tiene la siguiente forma:

$$
\boldsymbol{T}(y, \theta)=\left(\begin{array}{cccc}
\cos \theta & 0 & \sin \theta & 0 \\
0 & 1 & 0 & 0 \\
-\sin \theta & 0 & \cos \theta & 0 \\
0 & 0 & 0 & 1
\end{array}\right)
$$

Finalmente, la matriz de transformación homogénea que representa la transformación de rotación con respecto al eje $\mathrm{z}$ tiene la siguiente forma:

$$
\boldsymbol{T}(z, \theta)=\left(\begin{array}{cccc}
\cos \theta & -\sin \theta & 0 & 0 \\
\sin \theta & \cos \theta & 0 & 0 \\
0 & 0 & 1 & 0 \\
0 & 0 & 0 & 1
\end{array}\right)
$$

Por otro lado, en 1955, Denavit y Hartenberg demostraron que la representación más compacta de una transformación general entre dos articulaciones de un mecanismo requería cuatro parámetros que ahora son conocidos como los parámetros de Denavit y Hartenberg (parámetros D-H) y son el estándar para definir la geometría de un manipulador (Denavit, et al., 1955).

Para poder aplicar la convención de Denavit y Hartenberg es requerido que los sistemas de referencia asignados a los eslabones de un manipulador industrial satisfagan la condición de que el eje $x_{i}$ asignado a la articulación $i$ sea perpendicular e intersecte al eje $z_{i-1}$ asignado a la articulación $i-1$. Si esto se cumple, entonces solamente es requerido el uso de cuatro transformaciones para determinar la posición y orientación del sistema de referencia $i$ con respecto al sistema de referencia $i$ 1. En la figura 3, se muestran dos sistemas de referencia donde sus ejes $x_{i}$ y $z_{i-l}$ satisfacen la condición.

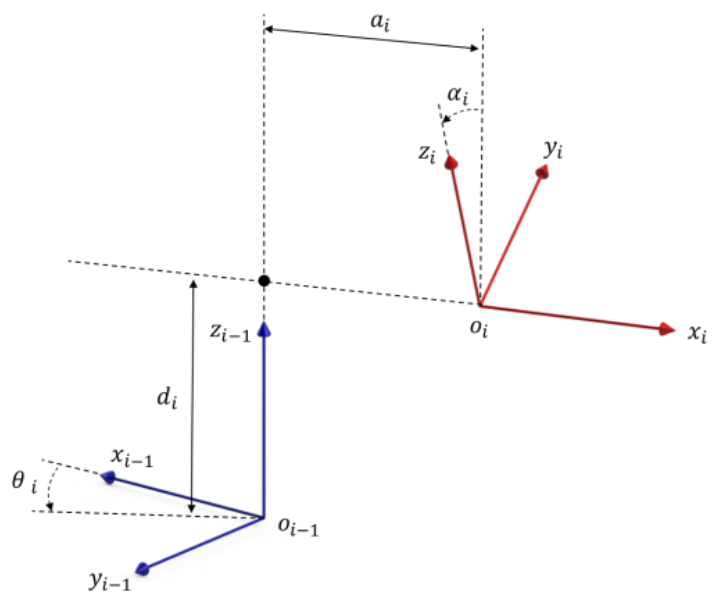

Figura 3: Sistemas de referencia que cumplen la condición de Denavit y Hartenberg.

Considerando la condición anterior entonces se cumple que al sistema de referencia $i$ fueron aplicadas las siguientes transformaciones homogéneas con respecto al sistema de referencia base, $i-1$ :

1. Rotación $\boldsymbol{T}\left(z, \theta_{i}\right)$, alrededor del eje $z_{i-1}$ un ángulo $\theta_{i}$, para obtener la orientación de $x_{i}$.

2. Traslación $\boldsymbol{T}\left(0,0, d_{i}\right)$, a lo largo del eje $z_{i-l}$ una distancia $d_{i}$. 
3. Traslación $\boldsymbol{T}\left(a_{i}, 0,0\right)$, a lo largo del eje $x_{i}$ una distancia $a_{i}$.

4. Finalmente, una rotación $\boldsymbol{T}\left(z, \alpha_{i}\right)$, alrededor del eje $x_{i}$ un ángulo $\alpha_{i}$.

Dado que el producto de matrices no es conmutativo, las transformaciones deben realizarse en el orden indicado, esto es:

$$
{ }^{i-1} T_{i}=T\left(z, \theta_{i}\right) T\left(0,0, d_{i}\right) T\left(a_{i}, 0,0\right) T\left(x, \alpha_{i}\right)
$$

Realizando el producto entre matrices finalmente se obtiene que la matriz que relaciona el sistema de referencia $i$ con respecto al sistema de referencia $i-1$ es:

$$
{ }^{i-1} T_{i}=\left(\begin{array}{cccc}
C \theta_{i} & -C \alpha_{i} S \theta_{i} & S \alpha_{i} S \theta_{i} & a_{i} C \theta_{i} \\
S \theta_{i} & C \alpha_{i} C \theta_{i} & -S \alpha_{i} C \theta_{i} & a_{i} S \theta_{i} \\
0 & S \alpha_{i} & C \alpha_{i} & d_{i} \\
0 & 0 & 0 & 1
\end{array}\right)
$$

Donde $\operatorname{C} \theta_{\mathrm{i}}=\operatorname{Coseno}\left(\theta_{\mathrm{i}}\right), \operatorname{S} \theta_{\mathrm{i}}=\operatorname{Seno}\left(\theta_{\mathrm{i}}\right), \operatorname{C} \alpha_{\mathrm{i}}=\operatorname{Coseno}\left(\alpha_{\mathrm{i}}\right)$ y $\operatorname{S} \alpha_{\mathrm{i}}$ $=\operatorname{Seno}\left(\alpha_{\mathrm{i}}\right)$.

Este método relaciona dos parámetros D-H con la longitud del eslabón y su torsión. El parámetro de longitud, $a_{i}$, se define como la distancia que existe entre los ejes de las articulaciones que contiene el eslabón y se mide a lo largo de una línea perpendicular a éstos, ver figura 4 . Por otro lado, el parámetro de torsión se define como el ángulo $\alpha_{\mathrm{i}}$ que existe entre los ejes de las articulaciones del eslabón y se determina mediante la proyección de los ejes en un plano que es perpendicular a la línea que permite obtener la longitud del eslabón. El sentido del ángulo de torsión se determina a partir del eje $i$ al eje $i+1$ aplicando la regla de la mano derecha.

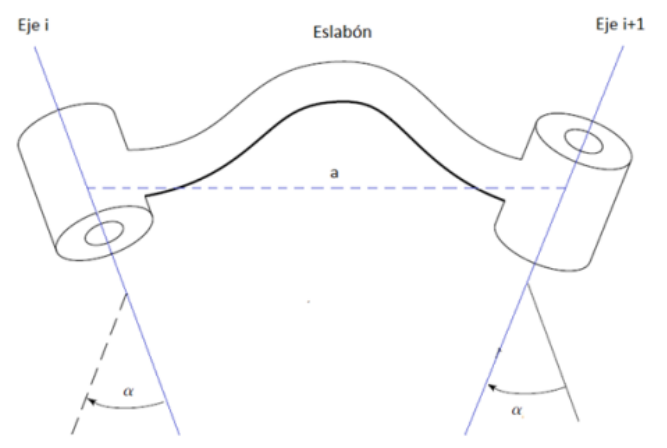

Figura 4: Parámetros geométricos de un eslabón.

De igual manera, el método considera dos parámetros D-H que indican la forma en la cual se conectan los eslabones para formar la cadena cinemática del robot, estos son el desplazamiento y el ángulo entre eslabones, ver figura 5. El desplazamiento, $d_{i}$, corresponde a la distancia que existe entre un eslabón y otro medido a lo largo del eje de la articulación en común. El ángulo entre eslabones, $\theta_{i}$, mide la rotación que presenta un eslabón con respecto a otro adyacente. Para cada tipo de articulación uno de estos dos parámetros es variable, en el caso de una articulación prismática el parámetro $d_{i}$ dependerá del desplazamiento que realice la articulación, y para las articulaciones rotacionales el parámetro $\theta_{i}$ especifica la rotación relativa de un eslabón con respecto a otro que se conectan mediante la articulación.

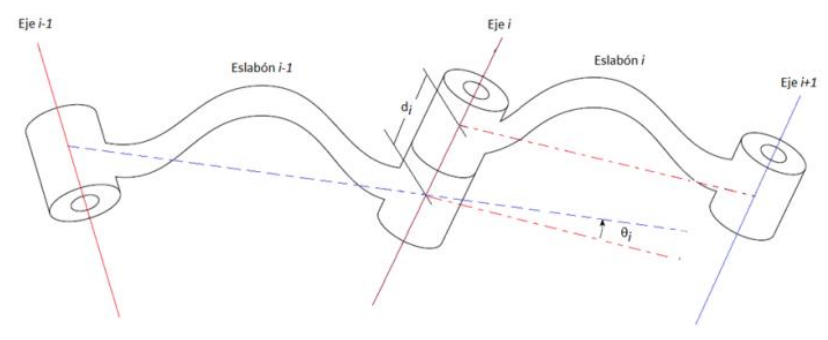

Figura 5: Parámetros de conexión entre eslabones.

Un ejercicio típico de cinemática directa se resuelve a través de seguir la siguiente secuencia:

1. Fijar los sistemas de referencia para todas las articulaciones y puntos del manipulador que lo requieran.

2. Calcular los parámetros D-H para cada articulación.

3. Calcular la matriz de transformación homogénea para cada par de articulaciones vecinas.

4. Multiplicar las matrices de transformación homogéneas obtenidas en el paso anterior para obtener la posición de cada articulación y del efector final con respecto a la base del manipulador.

En el paso 1 de la asignación de los sistemas de referencia se debe considerar las siguientes características:

- Asignar los ejes $z_{i}$ de cada sistema de referencia de los eslabones. El sentido positivo puede tomarse hacia cualquier dirección siempre y cuando se cumpla que:

- Cada eje $z_{i}$ se coloca sobre el eje de la articulación $i+1$ para $i=0$ hasta $n-1$.

- El último eje $z_{n}$ se coloca en el último eslabón del manipulador con la misma dirección que tenga el eje $z_{n-1}$.

- Fijar los orígenes $S_{i}$ de los sistemas de referencia:

- Fijar el origen del sistema de la base $S_{o}$ en cualquier punto del eje $z_{o}$ a partir del cual se determina la posición y orientación del elemento terminal del manipulador.

○ Fijar los orígenes de los sistemas de referencia $S_{i}$ solidario al eslabón $i$ para $i=1$ hasta $n$. Si los ejes $z_{i-1}$ y $z_{i}$ se cruzan se coloca el origen de $S_{i}$ en el punto de cruce. Si son paralelos o no coplanares situar el origen de $S_{i}$ sobre la intersección del eje $z_{i}$ con la línea perpendicular común a los ejes $z_{i-1}$ y $z_{i}$. 
- Asignar el sentido de los ejes $x_{i}$ e $y_{i}$ :

- Los ejes $x_{o}$ e $y_{o}$ se sitúan de modo que formen un sistema de referencia derecho con respecto al eje $z_{o}$, ver figura 6.

- Situar $x_{i}$ en la línea perpendicular común a $z_{i-1}$ y $z_{i}$ con la dirección del primero hacia el último para $i=1$ hasta $n$.

- Situar $y_{i}$ de modo que forme un sistema de referencia derecho con $x_{i}$ y $z_{i}$ para $i=1$ hasta $n$, ver figura 6 .

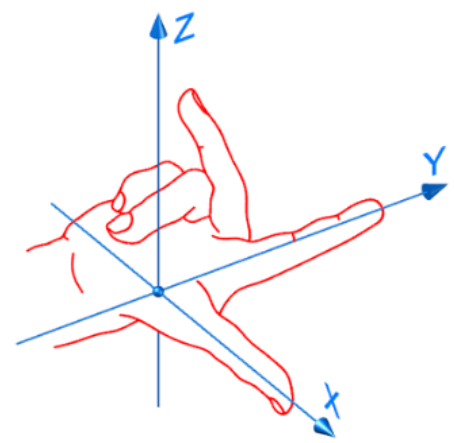

Figura 6: Regla de la mano derecha para definir sistemas de referencia derechos.

En el paso 2 los parámetros de D-H se obtienen de la siguiente manera:

- Obtener $\theta_{i}$ como el ángulo que hay que girar en torno a $z_{i-1}$ para que coincidan $x_{i-1}$ y $x_{i}$ para $i=1$ hasta $n$, ver figura $7 \mathrm{a}$.

- $\quad$ Obtener $d_{i}$ como la distancia medida a lo largo de $z_{i-1}$ que habría que desplazar $S_{i-1}$ para quedar alineado con $S_{i}$, para $i=1$ hasta $n$, ver figura 3 .

- Obtener $\alpha_{i}$ como el ángulo que habría que girar con respecto a $x_{i}$ para que coincidan $z_{i-1}$ y $z_{i}$ para $i=1$ hasta $n$, ver figura $7 \mathrm{~b}$.

- Obtener $a_{i}$ como la distancia medida a lo largo de $x_{i}$ que habría que desplazar $S_{i-1}$ para que coincida con $S_{i}$ para $i=1$ hasta $n$, ver figura 3 .

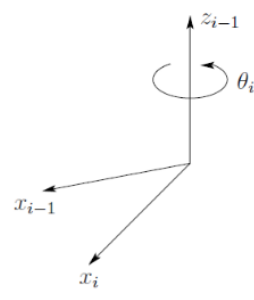

a) Parámetro $\theta_{i}$

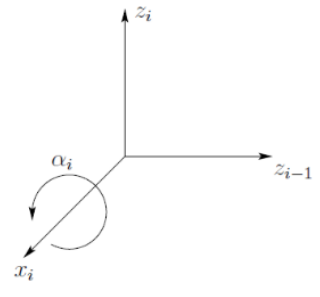

b) Parámetro $\alpha_{i}$
Figura 7: Determinación de los ángulos de DenavitHartenberg.
Es importante notar que la convención de Denavit - Hartenberg no da una definición única de los sistemas de referencia unidos a los eslabones del manipulador bajo los siguientes aspectos:

- Para el sistema de referencia de la base $S_{o}$ solo se especifica la ubicación del eje $z_{o}$, sin embargo, su origen y el sentido del eje $x_{o}$ puede elegirse de forma arbitraria.

- Cuando dos ejes son paralelos, la línea perpendicular común a ambos no se define de manera única, por lo tanto, el origen para estos sistemas tampoco es único.

- $\quad$ Cuando se cruzan los ejes $z_{i-1}$ y $z_{i}$ el sentido del eje $x_{i}$ es arbitrario.

- Cuando se tiene una junta prismática solo se puede determinar el sentido de su eje $z$, pero la ubicación de su origen es arbitraria.

Bajo estos casos, se puede aprovechar la indeterminación de forma que se simplifiquen las matrices de transformación homogéneas.

\section{Metodología}

El principal problema que presentan los estudiantes cuando se utiliza el método tradicional de enseñanza en un curso introductorio de robótica industrial es el poder construir el modelo cinemático del manipulador bajo estudio, esto se debe principalmente a que es necesario que tengan una muy buena comprensión de la configuración espacial del robot, incluyendo, en dónde se encuentran ubicadas las articulaciones en la estructura mecánica y qué tipo de movimientos es capaz de realizar el mecanismo, según los tipos de articulaciones, rotacional o prismática.

Por lo tanto, con el objetivo de facilitar la comprensión de este concepto, en esta sección se describe un procedimiento metodológico que brinda a los estudiantes la posibilidad de practicar con diferentes configuraciones de manipuladores de manera supervisada permitiendo reafirmar sus conocimientos a través de una plataforma desarrollada en MATLAB ${ }^{\mathrm{TM}}$ Simulink $^{\mathrm{TM}}$ utilizando la librería de SimMechanics ${ }^{\mathrm{TM}}$, los robots son modelados en Solidworks ${ }^{T M}$ y posteriormente exportados a SimMechanics $^{T M}$, además se evalúa la habilidad de los estudiantes para resolver un problema de cinemática directa de un manipulador físico construido con el equipo didáctico de LEGO Mindstorms ${ }^{T M}$, el cual es controlado mediante la librería de LEGO Mindstorms ${ }^{T M}$ EV3 para Simulink ${ }^{T M}$.

La metodología consiste en primer lugar en introducir los conceptos teóricos convenientes como la representación de la posición y orientación de un cuerpo rígido, las transformaciones homogéneas entre sistemas de referencia y el concepto de los parámetros $\mathrm{D}-\mathrm{H}$ para la obtención de la cinemática directa (conceptos presentados en la sección de preliminares del presente trabajo) a través de utilizar algunos ejemplos básicos de diferentes robots manipuladores.

Posteriormente, se introduce a los estudiantes la plataforma de simulación analizando los ejercicios de las configuraciones 
de robots vistos en la parte teórica; en este punto, es donde se refuerza el aprendizaje de una manera interactiva dentro del entorno de MATLAB ${ }^{\mathrm{TM}}$ Simulink $^{\mathrm{TM}}$, mediante la "Asignación de los sistema de referencia" a través de validar los parámetros D-H previamente determinados en el análisis teórico, visualizando el efecto de cada parámetro en la posición y orientación de cada sistemas de referencia.

En esta segunda etapa, se presentan al estudiante diferentes configuraciones de manipuladores que van cambiando su grado de complejidad al ir aumentando el número de grados de libertad de uno hasta cuatro, resolviendo configuraciones de robots que contienen únicamente articulaciones de tipo prismáticas o rotacionales, y finalmente robots con ambos tipos de articulaciones, ver figura 8.

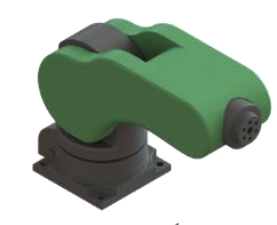

2 DOF CONFIGURACIÓN RP_2

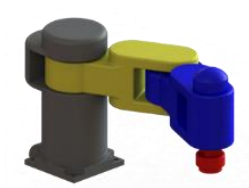

3 DOF CONFIGURACIÓN RRP_3

(Robot Scara)

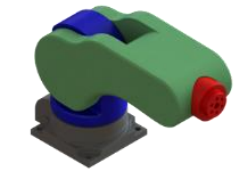

3 DOF CONFIGURACIÓN RRP_2

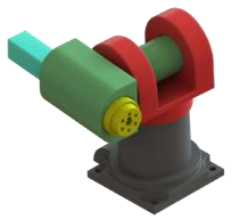

4 DOF CONFIGURACIÓN RRPR

(Robot Stanford)
Figura 8: Configuraciones utilizadas como ejercicios para estudiantes.

En la figura 9 se muestra un ejemplo de visualización en la plataforma de enseñanza de MATLAB $^{\mathrm{TM}}$ Simulink $^{\mathrm{TM}}$ correspondiente a un manipulador de 4 grados de libertad; el estudiante puede ver gráficamente la asignación de los sistemas de referencia de cada articulación conforme introduce el valor de los parámetros D-H y observar cómo es que ocurren los movimientos relativos entre los sistemas de referencia a medida que cambian los valores de las articulaciones durante la simulación. Esta es la primera herramienta que utiliza el estudiante para validar si se realizó correctamente la determinación de los parámetros $\mathrm{D}-\mathrm{H}$, ya que la orientación y posición de los sistemas de referencia de cada eslabón deberán coincidir con el obtenido en la parte teórica.

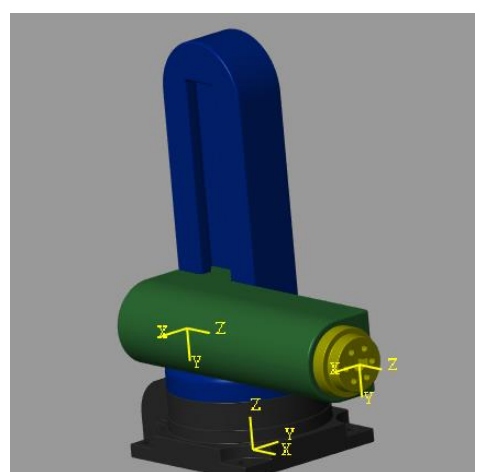

Figura 9: Manipulador de 4 grados de libertad en Simulink.
En la figura 10, se muestra la ventana de Simulink ${ }^{\mathrm{TM}}$ dónde el usuario introduce los valores deseados de las juntas articulares. El bloque de Simulink ${ }^{\mathrm{TM}}$ etiquetado con el nombre de Transformación Homogénea Experimental permite indicar el valor de los parámetros D-H para las cuatro transformaciones homogéneas descritas por la ecuación (5) y ver su posición y orientación en el modelo virtual mostrado en la figura 9.

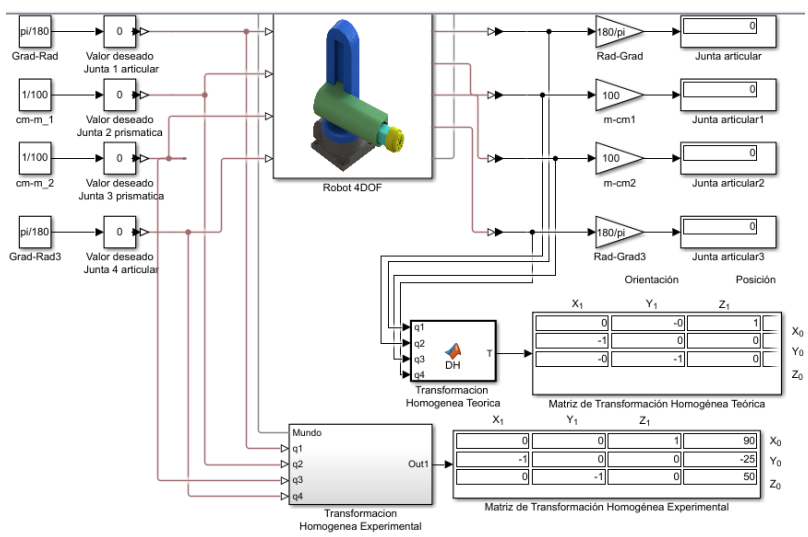

Figura 10: Interface de Simulink ${ }^{T M}$ para un robot de 4 grados de libertad.

La figura 11 muestra los elementos que se encuentran al interior del bloque, el valor de cada parámetro D-H obtenido en la etapa de análisis teórico, se introduce en el bloque correspondiente que representa la transformación de rotación o de traslación.

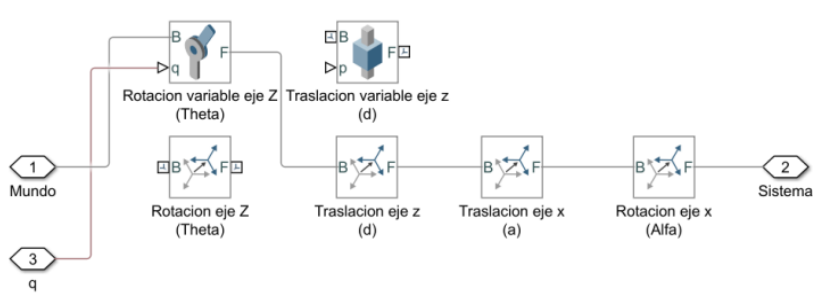

Figura 11: Elementos al interior del bloque de Transformación Homogénea Experimental

El cálculo de la matriz de transformación homogénea que representa la cinemática directa es realizado por los bloques mostrados en la figura 11 una vez que han sido colocados los valores de los parámetros D-H y se muestran en tiempo real durante la simulación en el bloque denominado Matriz de Transformación Homogénea Experimental (ver figura 10). Utilizando esta herramienta el estudiante puede validar que los parámetros D-H obtenidos son correctos si los sistemas de referencia mostrados en la simulación coinciden con los determinados en el análisis teórico (paso 2, del método descrito en la sección de preliminares para el cálculo de la cinemática directa); si lo anterior no se cumple, el alumno concluye que el error cometido corresponde a una asignación incorrecta de los parámetros D-H.

Posteriormente, en el bloque denominado Transformación Homogénea Teórica (ver figura 10), el estudiante introduce el código de un programa de MATLAB $^{\mathrm{TM}}$ que obtiene numéricamente el producto de matrices de transformación homogénea de la forma descrita por la ecuación (6), para determinar la posición y orientación del efector final con respecto a su base. Si el valor de la cinemática directa calculado 
por los bloques Transformación Homogénea Teórica y Transformación Homogénea Experimental son iguales, esto le indica al estudiante que realizó correctamente la determinación de la matriz de transformación homogénea (paso 3 y 4, del método descrito en la sección de preliminares para el cálculo de la cinemática directa); en caso contrario, si no coinciden el alumno concluye que su error es la obtención de la matriz homogénea.

El siguiente paso de la metodología corresponde a la evaluación de los conocimientos de los estudiantes en forma escrita tomando en cuenta tres aspectos: El primero es que realicen una asignación correcta de los sistemas de referencia para cada articulación y el efector final; el segundo es que identifiquen correctamente los parámetros D-H para cada articulación y el último aspecto es la obtención de la matriz de transformación homogénea.

La última etapa de la metodología consiste en una actividad experimental para determinar la cinemática directa de un robot físico construido con piezas de LEGO Mindstorms ${ }^{T M}$. Los estudiantes construyen un manipulador de dos grados de libertad, ver figura 12, utilizando una guía de ensamble.

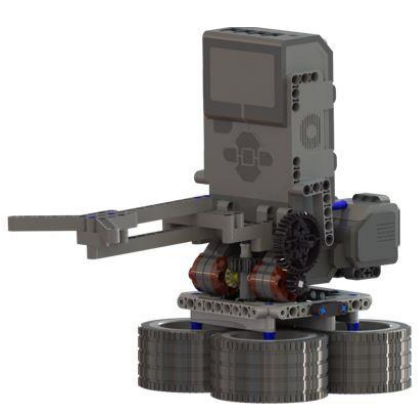

a) Modelo del robot

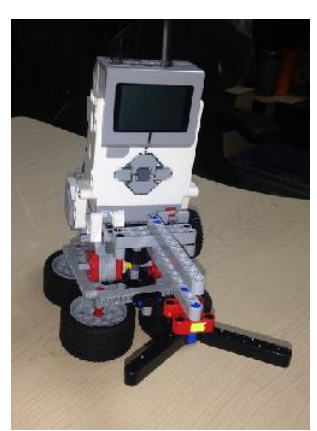

b) Robot físico
Figura 12: Manipulador con LEGO Mindstorms ${ }^{\text {TM }}$

Inicialmente, se definen valores angulares de prueba para cada una de las articulaciones y se obtiene la posición cartesiana mediante el análisis teórico, esto es, su cinemática directa. Posteriormente, utilizando el robot construido con piezas de LEGO Mindstorms ${ }^{T M}$ y su interfaz de control desarrollada en Simulink $^{T M}$ con ayuda de la librería de LEGO Mindstorms ${ }^{T M}$ EV3 que tiene MATLAB ${ }^{\mathrm{TM}}$, se realiza el movimiento de cada articulación a los valores angulares de prueba previamente establecidos, y se verifica si la posición del robot físico coincide con el cálculo teórico, ver figura 13.

Como un recurso adicional se proporciona al estudiante el archivo de simulación con el modelo del manipulador de LEGO Mindstorms $^{T M}$, ver figura 14, con el objetivo de que sea utilizado como soporte en caso de que en la primera iteración con el robot físico no obtenga el resultado correcto y de esta forma pueda utilizar la simulación como apoyo para realizar la actividad en forma correcta.

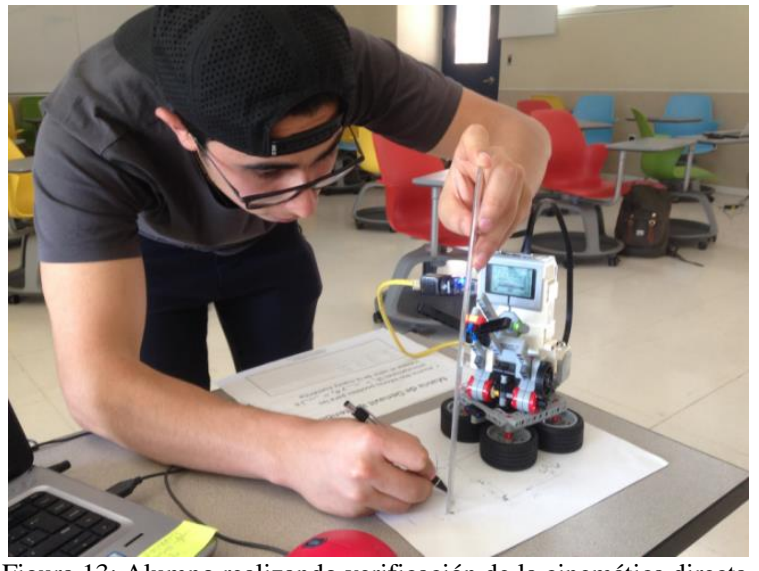

Figura 13: Alumno realizando verificación de la cinemática directa.

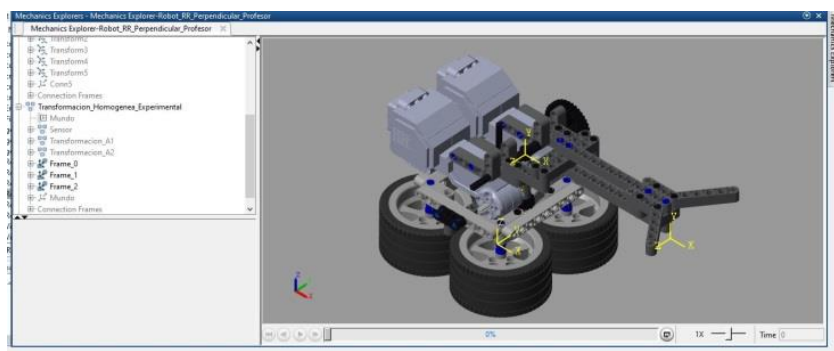

Figura 14: Simulación en MATLAB ${ }^{\mathrm{TM}}$ Simulink $^{\mathrm{TM}}$ del robot de LEGO Mindstorms $^{\mathrm{TM}}$

Dentro de los principales hallazgos encontrados al aplicar la presente metodología se pueden mencionar:

- La mayoría de los alumnos lograron cumplir la actividad en forma correcta y en la primera iteración.

- La principal dificultad que enfrentaron algunos estudiantes se dio al momento de elegir el sistema de referencia que coincidieran con la elección del diseñador para definir los ángulos positivos de las articulaciones.

- En muy pocos casos fue necesario que el profesor proporcionara apoyos adicionales para reforzar la parte conceptual para cumplir en forma exitosa con la actividad experimental con el robot físico con LEGO Mindstorms $^{T M}$.

\section{Conclusiones y trabajo futuro}

La actividad experimental propuesta para validar el aprendizaje de los alumnos de robótica de nivel licenciatura en el tema de cinemática directa y el nivel de cumplimiento de los alumnos permite inferir que la enseñanza de conceptos teóricos reforzada con simulaciones de modelos en $3 \mathrm{D}$ es una combinación muy adecuada para preparar a los estudiantes para resolver problemas de cinemática directa de manipuladores reales.

Se concluye que la plataforma de LEGO también se podría utilizar para analizar aspectos de diseño de manipuladores que vuelvan inviable la solución al problema de la cinemática directa utilizando la convención de Denavit y Hartenberg. 
El trabajo futuro será el realizar estudios del perfil de los estudiantes, así como un análisis cuantitativo de los resultados obtenidos. Finalmente, se considera también que es posible extender esta metodología a otros problemas como la cinemática inversa y la generación de trayectorias.

\section{English Summary}

\section{Teaching Forward Kinematics Using LEGO Mindstorms ${ }^{\text {TM }}$}

\begin{abstract}
The present work aims to show a methodology proposal for the teaching of forward kinematics of industrial manipulators, which is one of the main topics covered by an introductory course of robotics for university students. The technique used for teaching forward kinematics of manipulators is based on the convention introduced by Denavit and Hartenberg. This method has its foundations in the analysis of the rigid bodies that constitute the manipulator and the obtaining of parameters that allow the definition of the homogeneous transformation matrices that relate the frame of reference of the end-effector with the frame of reference of the base of the robot. The simulation and experimentation in the laboratory can be of great help to improve the understanding of these concepts.
\end{abstract}

The proposed approach allows students: Reaffirm their knowledge through a platform of modeled manipulators in Solidworks $^{\mathrm{TM}}$, exported to the MATLAB ${ }^{\mathrm{TM}}$ Simulink $^{\mathrm{TM}}$ environment using the SimMechanics ${ }^{\mathrm{TM}}$ library and, verify their ability to solve a problem of forward kinematics of a physical manipulator built with the LEGO Mindstorms ${ }^{\mathrm{TM}}$ controlled by the LEGO Mindstorms ${ }^{\mathrm{TM}}$ EV3 library for Simulink ${ }^{\mathrm{TM}}$.

Keywords: Forward Kinematics, MATLAB ${ }^{\mathrm{TM}}$, Simulink ${ }^{\mathrm{TM}}$, LEGO Mindstorms $^{\mathrm{TM}}$

\section{Agradecimientos}

Los autores desean agradecer el financiamiento otorgado por la iniciativa de proyectos de innovación educativa NOVUS del Tecnológico de Monterrey en su convocatoria del 2017, así como del apoyo del Tecnológico de Monterrey Campus Morelia, el Instituto Tecnologico Superior de Uruapan y la
Universidad Autónoma del Estado de México para el desarrollo del presente trabajo. Los autores también desean agradecer a los estudiantes que participaron durante la evaluación de la metodología del Tecnológico de Monterrey, Campus Morelia.

\section{Referencias}

Spong, Mark W. \& Vidyasagar, M., 2008, Robot dynamics and control, John Wiley \& Sons.

Corke, P. I., 2011, Robotics, visión \& control, Springer-Verlag, Berlin, Heidelberg.

Gil, A., O. Reinoso, J.M. Marin, L. Paya and J. Ruiz, 2014, Development and deployment of a new robotics toolbox for education, Comput Appl Eng. Educ., 1-12.

Joshi, S. S. , 2004, Development and implementation of a MATLAB simulation project for a multidisciplinary graduate course in autonomous robotics, Comput. Appl. Eng. Educ., Vol. 12, p.p. 54-64.

Miller, D., I. Nourbakhsh, and R. Siegwart, 2008 Robots for education Springer Handbook of Robotics, Springer-Verlag, Berlin, Heidelberg, p.p.,1283-1301.

Denavit, J., and R. S. Hartenberg, 1955, A kinematic notation for lower-pair mechanisms based on matrices, J Appl Mech 22, 215-221.

Othayoth, R., Rajeevlochana G. Chittawadigi, Ravi P. Joshi \& Subir K. Saha, 2017. Robot kinematics made easy using RoboAnalyzer software. Computer Applications in Engineering Education, vol. 25, no 5, p. 669680.

Gil, Arturo, Oscar Reinoso, José Maria Marin, Luis Paya, Javier Ruiz, 2014. Development and deployment of a new robotics toolbox for education. Computer Applications in Engineering Education, vol. 23, no 3, p. 443454.

Berenguel, M., Francisco rodríguez, José Carlos Moreno, José Luis Guzmán, Ramón González, 2015. Tools and methodologies for teaching robotics in computer science \& engineering studies. Computer Applications in Engineering Education, vol. 24,no 2, p. 202-214.

Indri, M., Lazzero, I. \& Bona, B., 2013. Robotics education: Proposals for laboratory practices about manipulators. Emerging Technologies \& Factory Automation (ETFA), 2013 IEEE 18th Conference on. IEEE. p. 1-8.

Barrientos, A., 2007. Fundamentos de robótica. Mc Graw-Hill. 